\title{
Safety and Prognostic Impact of Prophylactic Level VII Lymph Node Dissection for Papillary Thyroid Carcinoma
}

\author{
Ihab Samy Fayek ${ }^{1 *}$, Ahmed Ahmed Kamel ${ }^{2}$, Nevine FH Sidhom ${ }^{3}$
}

\begin{abstract}
Purpose: To study the safety of prophylactic level VII nodal dissection regarding hypoparathyroidism (temporary and permanent) and vocal cord dysfunction (temporary and permanent) and its impact on disease free survival. Materials and Methods: This prospective study concerned 63 patients with papillary thyroid carcinoma with N0 neck node involvement (clinically and radiologically) in the period from December 2009 to May 2013. All patients underwent total thyroidectomy and prophylactic central neck dissection including levels VI and VII lymph nodes in group A (31 patients) and level VI only in group B (32 patients). The thyroid gland, level VI and level VII lymph nodes were each examined histopathologically separately for tumor size, multicentricity, bilaterality, extrathyroidal extension, number of dissected LNs and metastatic LNs. Follow-up of both groups, regarding hypoparathyroidism, vocal cord dysfunction and DFS, ranged from 6-61 months. Results: The mean age was 34.8 and 34.3, female predominance in both groups with F: $M$ 24:7 and 27:5 in groups $A$ and B, respectively. Mean tumor size was 12.6 and $14.7 \mathrm{~mm}$. No statistical differences were found between both groups regarding age, sex, bilaterality, multicentricity or extrathyroidal extension. The mean no. of dissected level VI LNs was 5.06 and 4.72 and mean no. of metastatic level VI was 1 and 0.84 in groups $A$ and B, respectively. The mean no. of dissected level VII LNs was 2.16 and mean no. of metastatic LNs was 0.48. Postoperatively temporary hypoparathyroidism was detected in 10 and 7 patients and permanent hypoparathyroidism in 2 and 3 patients; temporary vocal cord dysfunction was detected in 4 patients and one patient, and permanent vocal cord dysfunction in one and 2 patients in groups $A$ and $B$, respectively. No significant statistical differences were noted between the 2 groups regarding hypoparathyroidism $(P=0.535)$ or vocal cord dysfunction $(P=0.956)$. The number of dissected LNs at level VI only significantly affected the occurrence of hypoparathyroidism $(<0.001)$ and vocal cord dysfunction $(<0.001)$.The DFS was significantly affected by bilaterality, multicentricity and extrathyroidal extension. Conclusions: Level VII nodal dissection is a safe procedure complementary to level VI nodal dissection with prophylactic central neck dissection for papillary thyroid carcinoma.
\end{abstract}

Keywords: Papillary thyroid carcinoma - prophylactic central neck dissection - level VI lymph nodes - level VII

Asian Pac J Cancer Prev, 16 (18), 8425-8430

\section{Introduction}

Papillary thyroid cancer (PTC) is the most common type of thyroid cancer accounting for about $80 \%$ of all thyroid cancers, and is the fifth leading malignancy in females (Cisco et al., 2012; Siegel et al., 2013).

Given the high rate of subclinical nodal metastases in PTC, many centers, have moved to routine prophylactic central nodal dissection (pCND) at the time of total thyroidectomy (TT) for all patients with PTC, pCND allows for more accurate assessment of nodal status, decreases the rate of local recurrence, reduces morbidity from reoperation if required, and may guide the dose of ablative postoperative radioiodine given (Mazzaferri EL Jhiang 1994; Scheumann et al., 1994; Hughes et al., 1996).

Dralle (2012) reports a significant risk of postoperative hypoparathyroidism after CND but suggests that pCND improves prognosis for papillary thyroid cancer Dralle (2013). Many studies showed an increased risk of recurrent paralysis in patients undergoing $\mathrm{CND}$, with rates of recurrent lesions ranging between $1 \%$ and $12 \%$ (Moo et al., 2009; Choi et al., 2010; Hughes and Doherty 2011). However, Tartaglia et al, 2014 reported no significant difference between TT alone or TT with CND. The American Thyroid Association's consensus statement on terminology of CND defines the innominate artery as the lower limit of a CND and this equates CND to level VI and the superior portion of level VII (ATA consensus, 2009).

Many studies highlighted the prognostic importance of additional dissection of level VII nodes as an integral and complementary component to Level VI neck nodes in pCND through the same cervical neck incision required for PTC (Choi et al., 2011; Wang et al., 2013; Fayek 2015), However only one study addressed the safety of the 
Ihab Samy Fayek et al

procedure regarding hypoparathyroidism and vocal cord dysfunction (Wang et al., 2013) requesting further studies to highlight and evaluate the previous results.

In this study, the author aims to study the safety of adding dissection of level VII nodes to the routine dissection of level VI nodes in pCND for PTC regarding hypoparathyroidism (temporary and permanent) and vocal cord dysfunction (temporary and permanent) and its impact on disease free survival.

\section{Materials and Methods}

This is a prospective study of 63 patients with papillary thyroid carcinoma (PTC) proved by Fine Needle Aspiration Cytology (FNAC) from December 2009 to May 2013. All patients had N0 neck nodes clinically and by Hi-Res neck ultrasound. All patients underwent Total Thyroidectomy (TT) and Central Neck Dissection (CND) in the form of dissection of levels VI and VII lymph nodes (LNs) in group A (31 patients) and level VI only in group B (32 patients). Level VI dissection was defined as including the prelaryngeal, pretracheal, and paratracheal nodes between the hyoid bone and the suprasternal notch. Level VII dissection was defined as the pretracheal and paratracheal superior mediastinal lymph nodes between the suprasternal notch and the innominate artery. Level VII dissection without opening the sternum was performed through the same collar neck incision for thyroidectomy, dissecting the anterior superior mediastinal lymph nodes above the innominate artery and vein (Figure 1a, b). For group A, the thyroid gland, level VI as well as level VII nodes were submitted each separately for histopathological examination. For group B, the thyroid gland and level VI nodes were also submitted each separately for histopathological examination. Patients' demographics, tumor size, multicentricity, bilaterality, extrathyroidal extension, number of dissected nodes and number of metastatic nodes in each level separately were recorded and analyzed.

Postoperatively serial measurements of serum calcium levels were recorded, defining hypocalcemia due to temporary hypoparathyroidism as a serum calcium

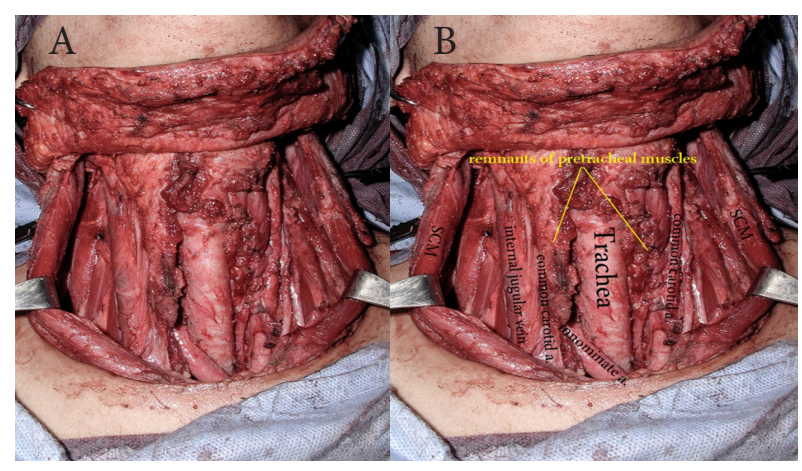

Figure 1.A) Operative field after Total Thyroidectomy and complete dissection of Levels VI and VII neck nodes B) Operative field after Total Thyroidectomy and complete dissection of Levels VI and VII neck nodes (Tagged)

Table 1. Patients' Sex and Tumor Characteristics

\begin{tabular}{lccc}
\hline & \multicolumn{3}{c}{ Group } \\
\cline { 2 - 4 } & A & B & P-value \\
\hline Sex & 24 & 27 & \\
$\mathrm{~F}$ & $77.40 \%$ & $84.40 \%$ & \\
$\mathrm{M}$ & 7 & 5 & 0.482 \\
& $22.60 \%$ & $15.60 \%$ & \\
Multicentricity & & & \\
Negative & 18 & 24 & \\
& $58.10 \%$ & $75.00 \%$ & \\
Positive & 13 & 8 & \\
& $41.90 \%$ & $25.00 \%$ & \\
Bilaterality & & & \\
Negative & 22 & 28 & 0.154 \\
& $71.00 \%$ & $87.50 \%$ & \\
Positive & 9 & 4 & \\
& $29.00 \%$ & $12.50 \%$ & 0.859 \\
Extrathyroidal extension & & \\
Negative & 21 & 21 & \\
Positive & $67.70 \%$ & $65.60 \%$ & \\
& 10 & 11 & \\
& $32.30 \%$ & $34.40 \%$ & \\
\hline
\end{tabular}

Table 2. Age, tumor size, and number of dissected and metastatic LNs in both groups

\begin{tabular}{|c|c|c|c|c|c|c|}
\hline & \multicolumn{6}{|c|}{ Group } \\
\hline & \multicolumn{6}{|c|}{ A } \\
\hline & Mean & Std. Deviation & Median & Minimum & Maximum & \\
\hline Age & 34.8 & 8.5 & 35 & 20 & 51 & \\
\hline tumor size (mm.) & 12.6 & 4.7 & 12 & 5 & 24 & \\
\hline Level VI LNs (dissected) & 5.06 & 1.77 & 5 & 2 & 8 & \\
\hline Level VI LNs (positive) & 1 & 1.13 & 1 & 0 & 3 & \\
\hline Level VII LNs (dissected) & 2.16 & 1.07 & 2 & 1 & 5 & \\
\hline \multirow[t]{2}{*}{ Level VII LNs (positive) } & 0.48 & 0.63 & 0 & 0 & 2 & \\
\hline & \multicolumn{5}{|c|}{ B } & \\
\hline tumor size (mm.) & Mean & Std. Deviation & Median & Minimum & Maximum & $\mathrm{p}$-value \\
\hline Level VI LNs (dissected) & 34.3 & 7.6 & 34.5 & 22 & 49 & 0.767 \\
\hline Level VI LNs (positive) & 14.7 & 5.4 & 14.5 & 7 & 24 & 0.148 \\
\hline Level VII LNs (dissected) & 4.72 & 1.85 & 5 & 2 & 8 & 0.423 \\
\hline Level VII LNs (positive) & 0.84 & 1.02 & 0.5 & 0 & 3 & 0.611 \\
\hline
\end{tabular}


Safety and Prognostic Impact of Prophylactic Level VII Lymph Node Dissection for Papillary Thyroid Carcinoma

concentration of less than $8 \mathrm{mg} / \mathrm{dL}$ at any point after operation up to 6 months postoperatively. Whereas permanent hypoparathyroidism was defined as the ongoing need for calcium and/or vitamin D supplementations for more than 6 months postoperatively to maintain normal serum calcium levels. All patients underwent routine preoperative and postoperative laryngoscopic evaluation.

Temporary vocal cord dysfunction (VCD) was

Table 3. Incidence of Hypoparathyroidism in both groups

\begin{tabular}{|c|c|c|c|}
\hline & Group & Group & P-value \\
\hline & A & B & \\
\hline \multicolumn{4}{|c|}{ Hypoparathyroidism } \\
\hline \multirow[t]{2}{*}{ No } & 19 & 22 & \\
\hline & $61.30 \%$ & $68.80 \%$ & \\
\hline \multirow[t]{2}{*}{ Yes } & 12 & 10 & \\
\hline & $38.70 \%$ & $31.30 \%$ & 0.535 \\
\hline \multirow[t]{2}{*}{ Total } & 31 & 32 & \\
\hline & $100.00 \%$ & $100.00 \%$ & \\
\hline
\end{tabular}

Table 4. Incidence of VCD in both groups

\begin{tabular}{|c|c|c|c|}
\hline & Group & Group & P-value \\
\hline & A & $\mathrm{B}$ & \\
\hline \multicolumn{4}{|c|}{ Vocal cord dysfunction } \\
\hline \multirow[t]{2}{*}{ No } & 26 & 27 & \\
\hline & $83.90 \%$ & $84.40 \%$ & \\
\hline \multirow[t]{2}{*}{ Yes } & 5 & 5 & \\
\hline & $16.10 \%$ & $15.60 \%$ & 0.956 \\
\hline \multirow[t]{2}{*}{ Total } & 31 & 32 & \\
\hline & $100.00 \%$ & $100.00 \%$ & \\
\hline
\end{tabular}

defined as sluggish or absent vocal cord motility on direct laryngoscopy that resolved subsequently within 6 months postoperatively. Permanent vocal cord dysfunction was defined as the decrease or absence of vocal cord motility on direct laryngoscopy persisting for 6 months or more postoperatively.

Preoperative and postoperative laryngoscopy was done at the otorhinolaryngology department at Cairo University hospital.

Follow-up of all patients in both groups extended up to 61 months.

Data was analyzed using IBM SPSS advanced statistics version 20 (SPSS Inc., Chicago, IL). Numerical data were expressed as mean and standard deviation or median and range as appropriate. Qualitative data were expressed as frequency and percentage. Chi-square test (Fisher's exact test) was used to examine the relation between qualitative variables. For quantitative data, comparison between two groups was done using MannWhitney test (non-parametric t-test). A P-value $<0.05$ was considered significant.

\section{Results}

Female: Male ratio was 24:7 and 27:5 in group A and $\mathrm{B}$ respectively $(\mathrm{P}=0.482)$ [Table 1]. The age of patients in group A ranged from 20-51 years (mean 34.8) and from 22-49 (mean 34.3) in group $\mathrm{B}(\mathrm{P}=0.767)$. Tumor size ranged from 5-24 $\mathrm{mm}$. in maximum diameter (mean 12.6) and 7-24 mm. (mean 14.7) in group A and $\mathrm{B}$ respectively $(\mathrm{P}=0.148)$ [Table 2]. Multicentricity was found in $13(41.9 \%)$ and $8(25 \%)$ patients in group A and $\mathrm{B}$ respectively $(\mathrm{P}=0.154)$. Bilaterality was detected in $9(29 \%)$ and $4(12.5 \%)$ patients in group A and B

Table 5. Relation between PO hypoparathyroidism and No. of dissected LNs in both groups

\begin{tabular}{lcccccccr}
\hline & \multicolumn{10}{c}{ Hypoparathyroidism } \\
\hline & Group & N & Mean & Std. Deviation & N & Mean & Std. Deviation & P-value \\
Level VI LNs (dissected) & A & 19 & 4.32 & 1.57 & 12 & 6.25 & 1.42 & 0.003 \\
Level VII LNs (dissected) & B & 22 & 3.73 & 1.16 & 10 & 6.9 & 0.99 & $<0.001$ \\
& A & 19 & 2.05 & 1.03 & 12 & 2.33 & 1.15 & 0.484 \\
\hline
\end{tabular}

Table 6. relation between PO VCD and No. of dissected LNs in both groups

\begin{tabular}{lccccccrr}
\hline & \multicolumn{10}{c}{ Vocal cord dysfunction } \\
\hline & Group & N & Mean & Std. Deviation & N & Mean & Std. Deviation & P-value \\
Level VI LNs (dissected) & A & 26 & 4.69 & 1.67 & 5 & 7 & 0.71 & 0.004 \\
Level VII LNs (dissected) & B & 27 & 4.22 & 1.55 & 5 & 7.4 & 0.55 & $<0.001$ \\
& A & 26 & 2.19 & 0.94 & 5 & 2 & 1.73 & 0.387 \\
\hline
\end{tabular}

Table 7. Correlation between tumor size ( $\mathrm{mm}$.) and LN metastasis in both groups

\begin{tabular}{|c|c|c|c|c|c|c|c|}
\hline \multirow{3}{*}{ Group } & \multicolumn{6}{|c|}{ LN metastasis } & \multirow[b]{3}{*}{ P-value } \\
\hline & \multicolumn{3}{|c|}{ Negative } & \multicolumn{3}{|c|}{ Positive } & \\
\hline & $\mathrm{N}$ & Mean & Std. Deviation & $\mathrm{N}$ & Mean & Std. Deviation & \\
\hline $\mathrm{A}$ & 13 & 9.5 & 2.4 & 18 & 14.9 & 4.6 & $<0.001$ \\
\hline B & 16 & 13.1 & 3.6 & 16 & 16.3 & 6.4 & 0.138 \\
\hline
\end{tabular}


Table 8. DFS in Relation to Patients and Tumor Characteristics and Complications

\begin{tabular}{|c|c|c|c|c|}
\hline Factors & $\begin{array}{l}\text { Number } \\
\text { of cases }\end{array}$ & $\begin{array}{l}\text { Number } \\
\text { of failure }\end{array}$ & 3 yrs (\%) & P-value \\
\hline All & 63 & 16 & 76.5 & \\
\hline \multicolumn{5}{|l|}{ Group } \\
\hline A & 31 & 8 & 76.1 & \\
\hline $\mathrm{B}$ & 32 & 8 & 77.2 & 0.741 \\
\hline \multicolumn{5}{|l|}{ Age groups } \\
\hline$<45$ years & 57 & 15 & 76.4 & \\
\hline$=>45$ years & 6 & 1 & 80 & 0.725 \\
\hline \multicolumn{5}{|l|}{ Sex } \\
\hline Male & 12 & 4 & 70.7 & \\
\hline Female & 51 & 12 & 78.2 & 0.268 \\
\hline \multicolumn{5}{|l|}{ Tumor size } \\
\hline$<=1 \mathrm{~cm}$ & 19 & 0 & 100 & \\
\hline$>1 \mathrm{~cm}$ & 44 & 16 & 68.9 & 0.006 \\
\hline \multicolumn{5}{|l|}{ Multicentricity } \\
\hline Negative & 42 & 3 & 94.9 & \\
\hline Positive & 21 & 13 & 35.5 & $<0.001$ \\
\hline \multicolumn{5}{|l|}{ Bilaterality } \\
\hline Negative & 50 & 7 & 88 & \\
\hline Positive & 13 & 9 & 27.4 & $<0.001$ \\
\hline \multicolumn{5}{|c|}{ Extrathyroidal extension } \\
\hline Negative & 42 & 1 & 100 & \\
\hline Positive & 21 & 15 & 26.7 & $<0.001$ \\
\hline \multicolumn{5}{|l|}{ Hypothyroidism } \\
\hline No & 41 & 9 & 76.2 & \\
\hline Yes & 22 & 7 & 78.1 & 0.315 \\
\hline \multicolumn{5}{|c|}{ Vocal cord dysfunction } \\
\hline No & 53 & 12 & 79.3 & \\
\hline Yes & 10 & 4 & 61.7 & 0.337 \\
\hline
\end{tabular}

respectively $(\mathrm{P}=0.105)$. Extrathyroidal extension was detected in $10(32.3 \%)$ and $11(34.4 \%)$ patients in group $A$ and $B$ respectively $(\mathrm{P}=0.859)$ [Table 1]. Number of dissected level VI LNs ranged from 2-8 LNs in both groups with a mean of 5.06 and 4.72 in group $A$ and $B$ respectively $(\mathrm{P}=0.423)$. Number of metastatic level VI LNs ranged from 0-3 LNs in both groups with a mean of 1 and 0.84 in group $A$ and $B$ respectively $(\mathrm{P}=0.611)$. Number of dissected level VII LNs in group A ranged from 1-5 LNs (mean 2.16) and metastatic level VII LNs ranged from 0-2 LNs (mean 0.48) [Table 2]. 12 patients (38.7\%) (10 temporary and 2 permanent) in group $A$ and 10 patients (31.3\%) (7 temporary and 3 permanent) in group B showed hypoparathyroidism $(\mathrm{P}=0.535)$. For statistical purposes due to small sample size, both temporary and permanent hypoparathyroid patients were summed in each group for comparison [Table 3]. 5 patients (16.1\%) (4 temporary and 1 permanent) in group A and 5 patients (15.6\%) (3 temporary and 2 permanent) in group B showed VCD $(\mathrm{P}=0.956)$. For statistical purposes due to small sample size, both, patients with temporary and permanent VCD were summed in each group for comparison [Table 4]. The occurrence of either hypoparathyroidism or VCD was significantly affected by the No. of dissected level VI LNs only in both groups, while the dissected No. of level VII LNs in group A didn't affect the occurrence of neither of these 2 complications [Tables 5 and 6]. Tumor size significantly affected nodal metastases in group A $(\mathrm{P}<0.001)$, but not in group $\mathrm{B}(\mathrm{P}=0.138)$ [Table 7]. No other intraoperative or postoperative complications were recorded in both groups. The median disease free survival (DFS) for group A was 35 months while for group B it was 35.5 months. There was no statistically significant difference regarding DFS between both groups. Age, sex and the occurrence of hypoparathyroidism or VCD, all didn't significantly affect DFS. On the other hand, tumor size $>1 \mathrm{~cm}$, multicentricity, bilaterality and extrathyroidal extension significantly affected DFS [Table 8]. Inspite all 8 failure patients (Distant/Locoregional) in group A had metastatic level VI and VII lymph nodes, not all patients with metastatic level VI and level VII lymph nodes had failures. The same in group B, all 8 failure patients (Distant/Locoregional) in group B had metastatic level VI lymph nodes, but not all patients with metastatic level VI lymph nodes had failures.

\section{Discussion}

In 1956, Crile noted the anatomical continuity of level VI and level VII nodes Crile (1956), which was described later, by Grebe and Hay in 1996, as a lymphatic continuity between the neck and the superior mediastinum (Grebe and Hay, 1996).

Since level VI, which sits high up in the neck, as well as level VII, which is hidden behind the manubrium sterni and medial thirds of both clavicles, cannot be assessed accurately by ultrasound and CT with poor sensitivity ranging between 50 and $70 \%$ Mulla (2012), in addition, normal-sized, Level VII lymph nodes can still harbor macrometastatic disease (Wang et al., 2013), and since level VII nodes are an important and integral prognostic factor in papillary thyroid carcinoma (Choi et al., 2011; Wang et al., 2013; Fayek 2015); the dissection of those nodal groups is an important step in the management plan of PTC.

Putting in mind that $\mathrm{pCND}$ was advised by the European Thyroid Association, the British Thyroid Association, and the American Thyroid Association (Pacini et al., 2006; Grubbs et al., 2007) and its benefit in the accurate staging of the tumor, which may guide subsequent treatment and follow-up, in addition to decreasing the recurrence of PTC, improving disease-specific survival, and significantly reducing levels of serum thyroglobulin, increasing the rate of athyroglobulinemia (White et al., 2007) so pCND was done in all patients (including level VII in group A) of this study.

It should be remembered that the rate of permanent hypoparathyroidism and unintentional permanent nerve injury is higher when cervical lymph node dissection is performed with total thyroidectomy than with total thyroidectomy alone (White et al., 2007). In this study, the permanent hypoparathyroidism was documented in $6.5 \%$ for group A and $9.4 \%$ for group B patients which was much less than that reported by Tartaglia et al, 2014 $25.4 \%$ of patients who underwent TT + CND, within the range reported by White et al, 2007 0-14.3\% while Pereira et al., 2005; Choi et al., 2011; Popadich et al.,2011; Wang et al., 2013; and Lee et al., 2015 reported only 2\%,3\% $, 0.8 \%, 4.6 \%$ and $3.3 \%$ permanent hypoparathyroidism respectively. Temporary hypoparathyroidism was 
Safety and Prognostic Impact of Prophylactic Level VII Lymph Node Dissection for Papillary Thyroid Carcinoma

documented in $32.3 \%$ and $21.9 \%$ in group $\mathrm{A}$ and $\mathrm{B}$ of the current study respectively, coinciding with the findings of Lee et al, 2007 with $20.5 \%-48 \%$ incidence and Lee et al, 2015 with $36.6 \%$ of transient hypoparathyroidism; with a higher incidence than other studies $12.7 \%$ (Tartaglia et al., 2014), 6\% (Wang et al., 2013) and 9.7\% (Popadich et al., 2011) but sometimes as high as $60 \%$ (Pereira et al., 2005). In this study, the permanent VCD was documented in $3.2 \%$ in group $\mathrm{A}$ and $6.3 \%$ in group $\mathrm{B}$ which was within the reported range (0-12\%) in other studies (Choi et al., 2011; Hughes and Doherty, 2011; Popadich et al., 2011; Giordano et al., 2012; Wang et al., 2013; Tartaglia et al., 2014; Lee et al., 2015), while temporary VCD was reported to be $12.9 \%$ in group A and $9.4 \%$ in group B which was slightly higher than the reported range (0.4-9\%) in other studies (Pereira et al., 2005; Popadich et al., 2011; Wang et al., 2013; Tartaglia et al., 2014; Lee et al., 2015).

In this study, we couldn't prove a statistically significant difference between both groups regarding hypoparathyroidism and VCD. Two studies evaluated the complication rate of TT + pCND compared to TT alone and the only statistical difference between the 2 groups was in the transient hypoparathyroidism $(\mathrm{P}<0.02)$ (Sywak et al., 2006; Roh et al., 2007). Wang et al, 2013 also added level VII to CND with no increased risk of permanent complications of hypoparathyroidism or recurrent laryngeal nerve injury. Another finding in this study, that may point to the safety of level VII nodal dissection, is that the occurrence of either hypoparathyroidism or VCD was significantly affected by the No. of dissected level VI LNs only in both groups, while the dissected No. of level VII LNs in group Adidn't affect the occurrence of neither of these 2 complications [Tables 5 and 6].

Tumor size $>1 \mathrm{~cm}$. significantly affected nodal metastases in group A (levels VI and VII), but not in group B (level VI alone) which is considered complementary to the results of Choi et al., 2010 who concluded that tumor size $\geq 1.5 \mathrm{~cm}$. increased level VII nodal metastases significantly and that complications due to level VII LN dissection are not at a higher rate than level VI LN dissection alone, Both studies, revealed the prognostic impact of level VII nodal dissection especially when the tumor size is larger than $1 \mathrm{~cm}$. in PTC, in addition Roh et al, 2011 and Chen et al, 2015 documented that tumor size $>1 \mathrm{~cm}$. was a predictor of ipsilateral central lymph node metastasis (CLNM) recommending pCND in patients with a tumor size $>1 \mathrm{~cm}$. DFS was not statistically different between both groups [Table 8], as many studies comparing TT+ pCND versus TT alone didn't find a survival benefit between both groups (Sywak et al., 2006; Costa et al., 2009; Zuniga et al., 2009; So et al., 2012). The most recent controversy has been provided by Barczewski et al, 2013 who published the first paper in the literature showing a benefit not only for local recurrence (5.5\% vs $12.4 \%$ ) but also for specific disease survival (98\% vs 92.5\%) for patients with PTC having TT + pCND in comparison with those who had only TT, major bias in this study are its retrospective nature and that patients considered at risk in any group had RAI treatment.

An extensive review of the literature stated that no arguments favoring pCND as a universal rule for patients with PTC. The guidelines and consensus documents of the most important medical and surgical societies are in the direction of selecting subgroups of patients with high risk of recurrence for pCND, especially T3 or T4 tumors, multifocal/bilateral tumors and patients with BRAF V600E mutation detected in the preoperative setting. In the rest of PTC, which are the majority, TT must be considered an oncological proper treatment providing the best overall survival. Ramírez-Plaza (2015)

Further studies are needed to define the need of level VII in addition to level VI nodal dissection in selected subgroups of patients with PTC.

In conclusion, adding level VII nodal dissection as a complementary and integral part to level VI nodes for prophylactic CND in patients with PTC is a safe procedure regarding either hypoparathyroidism or VCD. The occurrence of these complications was related to the no. of dissected level VI LNs but not to level VII LNs. Tumor size $>1 \mathrm{~cm}$. significantly affects nodal metastases in both level VI and VII LNs.

\section{References}

American Thyroid Association Surgery Working, Carty S, Cooper D, et al (2009). Consensus statement on the terminology and classification of central neck dissection for thyroid cancer. Thyroid, 19, 1153-8.

Barczyński M, Konturek A, Stopa M, et al (2013). Prophylactic central neck dissection for papillary thyroid cancer. $\mathrm{Br} J$ Surg, 100, 410-8.

Chen Q, Zou X, Wei T, et al (2015). Prediction of ipsilateral and contralateral central lymph node metastasis in unilateral papillary thyroid carcinoma: a retrospective study. Gland Surg, 4, 288-94.

Choi J, Choi Y, Park Y, et al (2011). Experience and analysis of level VII cervical lymph node metastases in patients with papillary thyroid carcinoma. J Korean Surg Soc, 80, 307-12.

Choi Y, Yun J, Kook S, et al (2010). Clinical and imaging assessment of cervical lymph node metastasis in papillary thyroid carcinomas. World J Surg, 34, 1494-9.

Cisco R, Shen W, and Gosnell J (2012). Extent of surgery for papillary thyroid cancer: preoperative imaging and role of prophylactic and therapeutic neck dissection. Curr Treat Options Oncol, 13, 1-10.

Costa S, Giugliano G, Santoro L, et al (2009). Role of prophylactic central neck dissection in cN0 papillary thyroid cancer. Acta Otorhinolaryngol Ital, 29, 61-9.

Crile G (1956). the pattern of metastasis of carcinoma of the thyroid. Ann Surg, 143, 580-7.

Dralle H (2012). Postoperative hypoparathyroidism: central neck dissection is a significant risk factor, Chirurg, 83, 1082 .

Dralle H (2013). Prophylactic central lymph node dissection improves prognosis for papillary thyroid cancer. Chirurg, 84, 149.

Fayek I (2015). Prophylactic level VII nodal dissection as a prognostic factor in papillary thyroid carcinoma: a pilot study of 27 patients. Asian Pac J Cancer Prev, 16, 4211-4.

Giordano D, Valcavi R, Thompson G, et al (2012). Complications of central neck dissection in patients with papillary thyroid carcinoma: results of a study on 1087 patients and review of the literature. Thyroid, 22, 911-7.

Grebe S and Hay I (1996). Thyroid cancer nodal metastases: biologic significance and therapeutic considerations. Surg Oncol Clin N Am, 5, 43-63.

Grubbs E, Rich T, Li G, et al (2008). recent advances in thyroid 
Ihab Samy Fayek et al cancer. Curr Probl Surg, 45, 156-250.

Hughes C, Shaha A, Shah J, et al (1996). Impact of lymph node metastasis in differentiated carcinoma of the thyroid: a matched pair analysis. Head Neck, 18, 127-32.

Hughes D and Doherty G (2011). Central neck dissection for papillary thyroid cancer, Cancer Control, 18, 83-8.

Lee D, Oh K, Cho J, et al (2015). The benefits and risks of prophylactic central neck dissection for papillary thyroid carcinoma: prospective cohort study. Int J Endocrinol, 2015, 1-6.

Lee Y, Kim S, Kim S, et al (2007). Extent of Routine Central Lymph Node Dissection with Small Papillary Thyroid Carcinoma. World J Surg, 31, 1954-9.

Mazzaferri E and Jhiang S (1994). Long-term impact of initial surgical and medical therapy on papillary and follicular thyroid cancer. Am J Med, 97, 418-28

Moo T, Umunna B, Kato M, et al (2009). Ipsilateral versus bilateral central neck lymph node dissection in papillary thyroid carcinoma. Ann Surg, 250, 403-8.

Mulla M (2012). Central cervical lymph node metastases in papillary thyroid cancer: a systematic review of imagingguided and prophylactic removal of the central compartment. Clin Endocrinol, 76, 131-6.

Pacini F, Schlumberger M, Dralle H, et al (2006). European consensus for the management of patients with differentiated thyroid carcinoma of the follicular epithelium. Eur $J$ Endocrinol, 154, 787-803.

Pereira J, Jimeno J, Miquel J, et al (2005). Nodal yield, morbidity, and recurrence after central neck dissection for papillary thyroid carcinoma. Surg, 138, 1095-1100.

Popadich A, Levin O, Lee J, et al (2011). A multicenter cohort study of total thyroidectomy and routine central lymph node dissection for cN0 papillary thyroid cancer. J Surg, 150, 1048-57.

Ramírez-Plaza C (2015). Central neck compartment dissection in papillary thyroid carcinoma: An update. World J Surg Proced, 5, 177-186.

Roh J, Kim J and Park C (2011). Central lymph node metastasis of unilateral papillary thyroid carcinoma: patterns and factors predictive of nodal metastasis, morbidity, and recurrence. Ann Surg Oncol, 18, 2245-50.

Roh J, Park J and Park C (2007). Total thyroidectomy plus neck dissection in differentiated papillary thyroid carcinoma patients: pattern of nodal metastasis, morbidity, recurrence, and postoperative levels of serum parathyroid hormone. Ann Surg, 245, 604-10.

Scheumann G, Gimm O, Wegener G, et al (1994). Prognostic significance and surgical management of locoregional lymph node metastases in papillary thyroid cancer. World J Surg, 18, 559-68.

Siegel R, Naishadham D, Jemal A (2013). Cancer statistics. $C A$ Cancer J Clin, 63, 11-30.

So Y, Seo M and Son Y (2012). Prophylactic central lymph node dissection for clinically node-negative papillary thyroid microcarcinoma: influence on serum thyroglobulin level, recurrence rate, and postoperative complications. Surg, 151, 192-8.

Sywak M, Cornford L, Roach P, et al (2006). Routine ipsilateral level VI lymphadenectomy reduces postoperative thyroglobulin levels in papillary thyroid cancer. Surg, 140, 1000-5.

Tartaglia F, Blasi S, Giuliani A, et al (2014). Central neck dissection in papillary thyroid carcinoma: Results of a retrospective study. Int J Surg, 12, 57-62.

Wang L, Versnick M, Gill A, et al (2013). Level VII is an important component of central neck dissection for papillary thyroid cancer. Ann Surg Oncol, 20, 2261-5.
White M, Gauger P and Doherty G (2007). Central lymph node dissection in differentiated thyroid carcinoma. World J Surg, 31, 895-904.

Zuniga S and Sanabria A (2009). Prophylactic central neck dissection in stage N0 papillary thyroid carcinoma. Arch Otolaryngol Head Neck Surg, 135, 1087-91. 